\title{
A REVIEW: TERAHERTZ SPECTROSCOPY AS A VIABLE DYNAMIC TOOL FOR PROTEIN AND ORGANIC MOLECULES CHARACTERIZATION
}

\author{
Hidayatul Aini Zakaria* \\ Universiti Malaysia Terengganu, 21030 Kuala Terengganu, Terengganu, Malaysia \\ *Corresponding author: hidayatul@umt.edu.my
}

\begin{abstract}
THz spectroscopy is a relatively new spectroscopic method in which the THz useful range of frequencies is from $300 \mathrm{~cm}-1$ down to $3 \mathrm{~cm}-1$, a spectral region unachievable by FTIR spectroscopy. Multiple resonances in the absorption spectra of organic molecules exist in the THz range around $0.1-10 \mathrm{THz}$ (3 to $300 \mathrm{~cm}-1$ ) where these resonances arise from electromagnetic wave interaction of the THz source with the low-frequency, and hence large- scale, vibrational modes within the organic macromolecules. These major vibrational modes correspond to the quaternary or overall structure of macromolecules. The concerted motion of protein structure can also be investigated by $\mathrm{THz}$ spectroscopy, which enables both energy and momentum transfer at the picosecond time scale. The short bursts of far-IR radiation in an ultra-fast time scale in THz spectroscopy allow researchers to study the dynamic processes of various materials for a large variety of applications. This review paper discusses the probability of utilizing $\mathrm{THz}$ spectroscopy as a probe to determine the intermolecular structures of macromolecules as well as basic technical knowledge in $\mathrm{THz}$ spectroscopy.
\end{abstract}

(Keywords: Terahertz, spectroscopy, vibrational, proteins, organic molecules)

\section{INTRODUCTION}

Infrared (IR) spectroscopy is a classical chemical tool dating back to the early $20^{\text {th }}$ century (Hagen \& Rubens, 1902), whereas $\mathrm{THz}$ spectroscopy have only garnered serious attention about 20 years ago (Beard et al, 2002; Fattinger \& Grischkowsky, 1989). The effort to study THz spectroscopy was triggered by the challenge to produce and detect ultra-short electric transients (changes in electrical voltage) as they were propagated along an electric transmission line (Beard et al, 2002). These electrical pulses create very small bursts of electromagnetic radiation in the far-IR frequency wavelength from 0.2 to $2.0 \mathrm{THz}(6.66$ to $66.6 \mathrm{~cm}^{-1}$ ) (Fattinger \& Grischkowsky, 1989). $\mathrm{THz}$ spectroscopy, sometimes referred as T-ray spectroscopy (Markelz, 2008), allows direct interrogation of complex permittivity of samples spanning a broad bandwidth of $0.1-8.0 \mathrm{THz}$ (3.33$266.4 \mathrm{~cm}^{-1}$ ) (Shen et al, 2003) without the need for cryogens and simultaneously allowing extremely flexible sample control and optics arrangements (Markelz, 2008).

$\mathrm{THz}$ rays provides an safer alternative to $\mathrm{x}$-rays due to its non-ionizing radiative property for imaging purposes through cloth, skin, paper and many others imaging materials. The investigation of the $\mathrm{THz}$ fields has considerably grown with much emphasis concentrating on the $\mathrm{THz}$ spectroscopy of biological materials. Several investigations that have linked $\mathrm{THz}$ spectroscopy to the biological or biochemistry fields have been conducted by various groups of researchers worldwide, including (i) the analysis of low-frequency molecular vibration of DNA and RNA strands (Fischer et al, 2005; Mickan et al, 2002), (ii) several types of proteins including lysozyme, myoglobin, bacteriorhodopsin and viruslike particles (Falconer et al, 2010; Markelz et al, 2002; Png et al, 2009; Zakaria et al, 2011; Zhang $\&$ Durbin, 2006), (iii) the interaction between electromagnetic radiation and biological materials (Doria et al, 2004; Pickwell et al, 2004; Smye et al, 2001), (iv) the detection of potentially harmful biological agents (Brown et al, 2004) and, (v) the application of $\mathrm{THz}$ imaging to detect skin cancer (Pickwell et al, 2004; Woodward et al, 2003).

\section{THE TECHNICAL ASPECT OF THZ SPECTROSCOPY}

The rapid growth of interest in the $\mathrm{THz}$ field is largely due to the emergence of the technology needed to access the $\mathrm{THz}$ region, including the development of $\mathrm{THz}$ source and detectors in recent years (Plusquellic et al, 2007). Vanexter and coworkers developed the $\mathrm{THz}$ photoconductive transmitter and detector based on silicon technology (Vanexter \& Grischkowsky, 1990) spanning the frequency range of $0.1-2 \mathrm{THz}\left(3.33-66.6 \mathrm{~cm}^{-1}\right)$. In the last decade, photoconductive antennae technology was manipulated and engineered 
for the generation and detection of $\mathrm{THz}$ rays (Fattinger \& Grischkowsky, 1989; Grischkowsky et al, 1990). Most THz sources relied on inorganic semiconductor materials - gallium arsenide (GaAs), zinc telluride $(\mathrm{ZnTe})$, and indium arsenide (InAs). These semiconductors can be used to transform an ultra-short (around 100 fs) near infrared pulsed laser to a $\mathrm{THz}$ pulse by excitation of electron-hole pairs in a semiconductor structure (Davies et al, 2002). The higher photon energy of the near-infrared pulse over the semiconductor band gap (energy difference from the top of valence band to the bottom of the conduction band) will create electron hole-pairs close to the surface of the $\mathrm{THz}$ source. A THz pulse is generated by the photo-excited acceleration of electron-hole pairs of the semiconductor material when the near-IR laser pulse reaches the semiconductor surface (Davies et al, 2002).
The channel of field effect transistors may be utilized as resonators for plasma waves where the dimensions and gate length of of the plasma frequency of the resonator may reach the $\mathrm{THz}$ range (Knap \& Dyakonov, 2013) For THz pulse detection, GaAs (Lu et al, 1998), silicon (Si), and gallium nitride $(\mathrm{GaN})$ (Kachorovskii \& Shur, 2008) are typically used as plasma (THz) wave detectors. The $\mathrm{THz}$ detection mechanism is as follows (Dyakonov \& Shur, 1996): incoming $\mathrm{THz}$ radiation excites the plasma wave in the FET that is translated as a voltage drop across the FET structure. This voltage drop between the drain to the source of the FET is correlated with a resonance response from the incoming electromagnetic radiation. The electromagnetic radiation will then provide the spectra needed to study the intramolecular characteristic being studied where the theoretical calculations will be discussed in the following paragraphs.

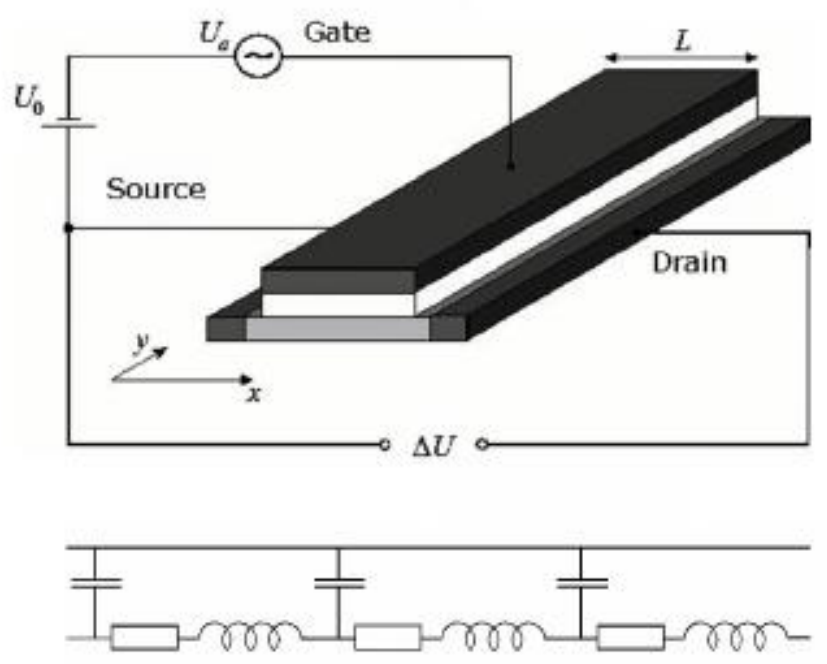

Figure 1. Schematics of a FET utilized as THz detector (upper image), and its corresponding equivalent circuit (lower image) (Knap \& Dyakonov, 2013)

In general, $\mathrm{THz}$ data measurements are obtained in the time domain (raw data) by recording the temporal field strength of a short electromagnetic pulse that lasts only for a few picoseconds. The Fast Fourier Transform (FFT) is the common algorithm used to convert these time domain signals (i.e., an estimate of the impulse response of the sample) to the frequency domain (Fischer, 2005b; Yin et al, 2007). From this conversion, the frequency response of the sample can be estimated. A THz pulse will be modified after propagating through a sample (dielectric material), because of the absorption and dispersion in the sample. By comparing this $\mathrm{THz}$ pulse with an appropriate reference pulse, an estimate can be obtained of the average absorption (absorption of EM waves by the sample) and the refraction (dispersion of light in the sample) from the sample measurements (Fischer 2005). 
Absorptions of electromagnetic radiation in the $\mathrm{THz}$ region are frequently related to hydrogenbonding interactions between separate molecules and variations in the crystal lattice (Fischer et al, 2007). THz data were acquired in the time domain by recording the temporal field strength of the short electromagnetic pulse. The following signal processing analysis is based on the $\mathrm{THz}$ studies of Bernd M Fischer (Fischer, 2005b).

The time domain signal, $\mathrm{j}(\tau)$ *where $\tau$ represents the time delay+ is a convolution of the electric pulse of the $\mathrm{THz}$ pulse $\mathrm{E}(\mathrm{t})$ with the $\mathrm{THz}$ detector pulse $\mathrm{D}(\mathrm{t})$ as given:

$$
j(t)=\frac{1}{T} \int_{0}^{T} E(t) D(t-\tau) d t
$$

The linear response of a material to a $\mathrm{THz}$ ray is determined by the dielectric properties of the material where the complete information of the dielectric function of that material is contained in its reflected THz pulse (Fischer, 2005b). Consider that an electromagnetic plane wave of frequency $\omega$ propagating through a sample in the $\mathrm{z}$-direction with the dielectric constant of $\hat{n}=n+i k$ will produce a time dependent electric field as follows:

$$
\begin{array}{r}
E(z, t)=E_{O}(t) \exp \left\{i\left(\omega t+\frac{\widehat{n \omega}}{c} z\right)\right\}=E_{0}(t) \exp \\
\left\{i \frac{n \omega}{c} z\right\} \cdot \exp \left\{-\frac{\kappa \omega}{c} z\right\}
\end{array}
$$

Here, the waveform before propagation through the sample is represented by the time dependent term $\mathrm{E}_{0}(t)=\mathrm{E}_{0} \mathrm{e}^{\mathrm{iwt}}$.

A THz reference pulse propagating through vacuum is given by:

$$
E_{\text {ref }}=E_{0}(\omega) \exp \left\{i \omega \frac{d}{c}\right\}
$$

Consider now a $\mathrm{THz}$ pulse after propagating through a dielectric material of thickness $\mathrm{d}$. A THz pulse will be modified after propagating through a sample (dielectric material), because of the absorption and dispersion in the sample. Thus, the frequency dependent field after the $\mathrm{THz}$ pulse propagation through a material is given by:

$E_{\text {sam }}(\omega)=E_{0}(\omega) \exp T\left\{i \frac{n(\omega) \omega}{c} d\right\} \cdot \exp \left\{-\frac{\kappa(\omega) \omega}{c} d\right\}$

Where $\mathrm{T}$ is the Fresnel losses at the interface of the sample (Bea \& Teich, 1974):

$\mathrm{T}=\frac{4 n(\omega)}{(n(\omega)+1)^{2}}$
By dividing this $\mathrm{THz}$ sample pulse with an appropriate $\mathrm{THz}$ reference pulse, an expression for the frequency dependent ratio (complex refractive index) can be obtained:

$$
\begin{aligned}
\frac{E_{\text {sam }}(\omega)}{\operatorname{Eref}(\omega)} & =A e^{i \Phi} \\
& =\frac{4 n(\omega)}{(n(\omega)+1)^{2}} \exp \left[-\alpha(\omega) \frac{d}{2}+i n(\omega) 2 \pi \frac{d}{c}\right]
\end{aligned}
$$

The absorption of light by an optical medium can be quantified by a parameter called the absorption coefficient, $\alpha$; the index of refraction, $\mathrm{n}$, can be simplified as the ratio of the vel ocity of light in free space to the velocity of light in a medium. The estimation of the average absorption coefficient (energy propagation through homogenous system) can be derived from the imaginary part of Equation [3.6], as shown in the equation below:

Absorption coefficient, $\alpha(\omega)=-\frac{2}{d} \ln \frac{(n(\omega)+1)^{2}}{4 n(\omega)} A$

where $\mathrm{A}$ is the complex amplitude.

The index of refraction (change in direction of wave propagation) can be derived from the real part of Equation [3.6] as shown in Equation [3.8]:

$$
n(\omega)=1+\frac{\Phi c}{w d}
$$

A schematic diagram of the $\mathrm{THz}$ spectroscopy system is illustrated in Figure 2. Measurements are typically conducted in a nitrogen-purged environment to reduce water vapour absorption by the $\mathrm{THz}$ radiation (Fischer, 2005a). An ultra-short (typically around $100 \mathrm{fs}$ ) laser pulse from the visible or near-IR laser (around $800 \mathrm{~nm}$ ) is divided into 2 directions by a beam splitter (Picometrix, 2005). One laser pulse is directed to a $\mathrm{THz}$ emitter, which transforms the incident laser pulse into a THz pulse; the other pulse serves as a detector monitoring the temporal shape of the radiated $\mathrm{THz}$ waveform (Walther et al, 2002). A sample is deposited in the propagation path of the $\mathrm{THz}$ wave between the $\mathrm{THz}$ emitter and the detector. The sample is irradiated by the $\mathrm{THz}$ wave from the $\mathrm{THz}$ emitter, and the transmitted $\mathrm{THz}$ pulse is detected by the $\mathrm{THz}$ detector. The detected signal from the $\mathrm{THz}$ detector is then transformed by Fourier transform and the complex valued spectra of the $\mathrm{THz}$ wave is obtained (Fischer, 2005a). Far-IR optical properties (absorption coefficient and index of refraction) of the sample can be determined as a function of frequency from the resolved $\mathrm{THz}$ spectra (Ferguson \& Zhang, 2002). 


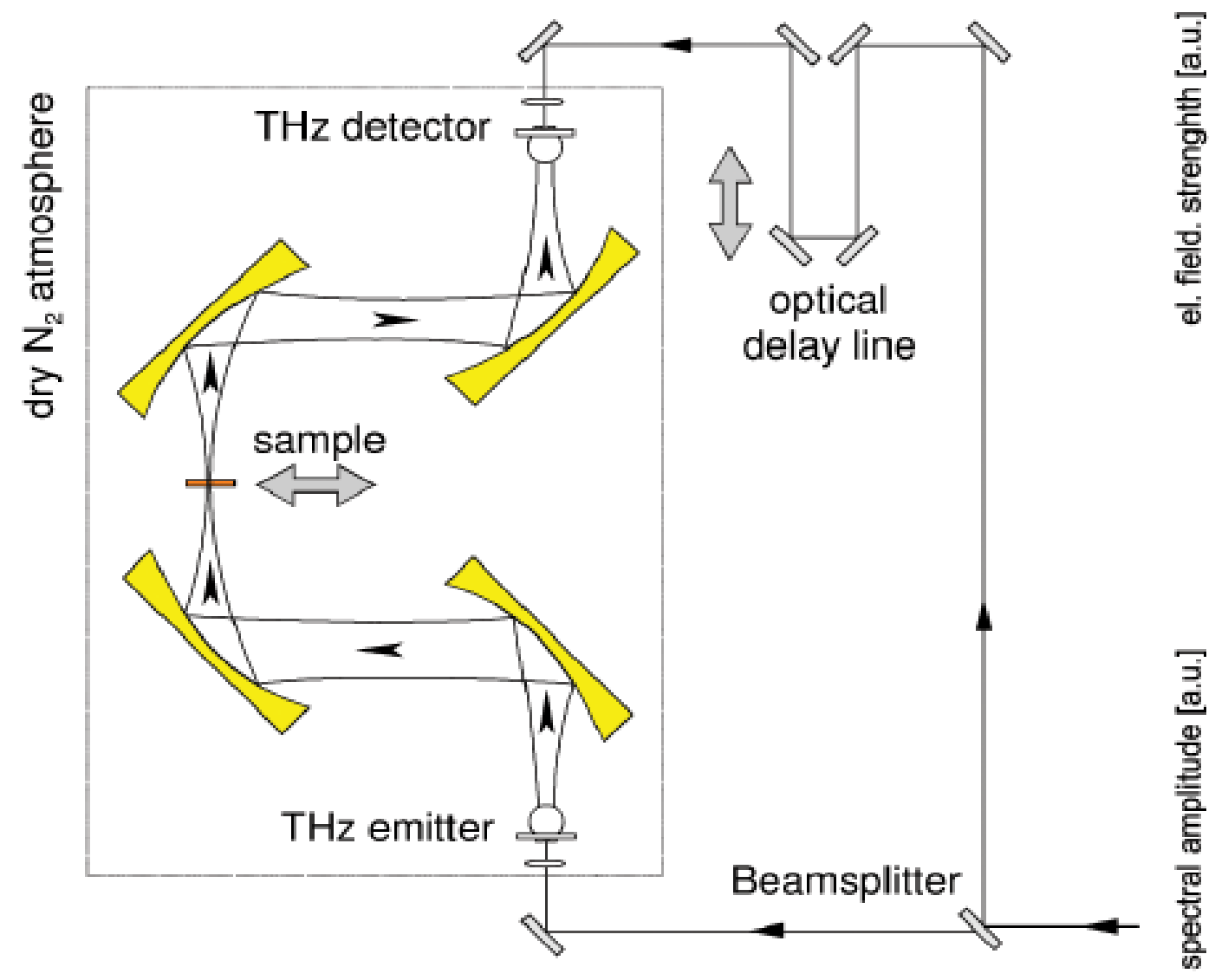

Figure 2. Schematic diagram of THz spectroscopy system adopted from Fischer (Fischer et al, 2007)

$\mathrm{THz}$ spectroscopy has several notable advantages over other forms of spectroscopic methods. Vibrational modes in the $\mathrm{THz}$ region are more widespread and include motions of all of the atoms in the molecular structure, whereas vibrational modes in the mid-IR wavelength are typically located within specific sites in the molecular conformation (Plusquellic et al, 2007). THz radiation has a longer wavelength than the near infra-red radiation, thus it will provide sharper images with better spatial resolution because it is less affected by Rayleigh scattering than near-infrared radiation (Beard et al, 2002). One of the many advantages of $\mathrm{THz}$ spectroscopy

over far-IR spectroscopy is that far-IR spectroscopy uses blackbody radiation sources and phonon detectors, while $\mathrm{THz}$ spectroscopy incorporates bright sources and sensitive detectors that can provide a higher signal-to-noise ratio and a wider dynamic range than far-IR spectroscopy (Grischkowsky et al, 1990). Another advantage of $\mathrm{THz}$ spectrometry is that it allows time-resolved far-IR spectroscopy to cover the spectral range of $0.1-20 \mathrm{THz}(3-600 \mathrm{~cm}-$ 1) in an ultra-fast time scale that provides sub-picosecond temporal resolution (Beard et al, 2002). Vivid changes in nucleic acid and component protein properties were observed when they were inserted into chromosomes or functional membranes (Sauer, 1995), which might be analysed using the picosecond time scale technology of THz spectroscopy.

There has been a notable increase in the interest in exploring the low-frequency collective vibrational modes within large biomolecules because these modes might provide valuable information about the conformational state of the biomolecules (Brandt et al, 2008). These low-frequency collective structural vibrational modes consisting of concerted largescale motions of biomolecules dictated by biomolecule architecture were believed to bear crucial roles towards biomolecular function and conformational modification changes (Markelz, 2008). However, 
spectroscopic studies in the low-frequency spectral region were often limited by technological constraints, such as insufficient light source power and detector sensitivity (Fischer, 2005b), owing to which it was difficult to resolve both energy and momentum transfers in the picosecond time scale (He et al, 2011b). THz spectroscopy with its inherent ability to probe the intra-molecular structure of materials in the low frequency spectral region. $1-8.0 \mathrm{THz}(3.33-266.4 \mathrm{~cm}-1 \mathrm{might}$ fill in this gaping research gap.

\section{ANALYSIS OF ORGANIC MOLECULES BY THZ SPECTROSCOPY}

Advancements in $\mathrm{THz}$ technology have enabled studies by $\mathrm{THz}$ spectroscopy for the characterisation of very far-IR vibrational modes of chemical compounds (Ueno \& Ajito, 2008). This intermolecular vibrational mode includes low-frequency vibrations around the hydrogen bonds (Walther et al, 2002), phonon modes that are IR-active intermolecular modes in a crystalline sample (Siegrist et al, 2006), and low-frequency delocalised modes that consist of the samples' entire vibrational motions (Giraud et al, 2003). THz spectroscopy provides the means to actively investigate the quaternary structure of organic molecules by the interaction of $\mathrm{THz}$ radiation with low-frequency vibrational modes of organic molecules. The dielectric functions of the organic molecules benzoic acid and its monosubstituted derivatives 2-, 3- and 4-hydroxybenzoic acid in pressed pellet form were investigated at $0.5-4.5 \mathrm{THz}\left(17-150 \mathrm{~cm}^{-1}\right)$ using $\mathrm{THz}$ time domain spectroscopy by Walther and co-workers at the University of Freiburg, Germany. Despite similarities in the molecular structure, the absorption spectra of these biomolecules had very little in common (Walther et al, 2002).

The high sensitivity of $\mathrm{THz}$ vibrational modes to small changes in the overall biomolecular structure in addition to the hydrogen bonding environment were evidenced again in a THz study of the enantiomeric crystalline structure of L-, D-, and DL-alanine (Yamaguchi et al, 2005) and the pure enantiomers and racemic compounds of tartaric acid (Fischer et al, 2007). Recently, the absorption spectra of organic crystals diglycine lithium nitrate, triglycine zinc chloride, and diglycine hydrobromide have shown clear resonant behavior in the permittivity diagram with sharp absorption peaks at $32.11 \mathrm{~cm}^{-1}$ ,44.16 $\mathrm{cm}^{-1}$, and $51.72 \mathrm{~cm}^{-1}$ (Trzebiatowska- Gusowska et al, 2014).

Comparisons of hydrated and dehydrated tri-alanine samples caused calculative differences in its respective $\mathrm{THz}$ spectra, demonstrating that use of a hydrogen-bonded solvent has a huge effect on the intermolecular structure of organic molecules investigated in the $\mathrm{THz}$ spectral range (Siegrist et al, 2006). A study of the intermolecular characteristics of chlorobenzene in the $\mathrm{THz}$ region conducted by Fischer (2007) showed that at a higher temperature $(295 \mathrm{~K})$, the absorption spectrum of chlorobenzene was swamped by large water absorption. In contrast, at a lower temperature (100 $\mathrm{K})$, the constitution of chlorobenzene changed from liquid to crystalline; hence, a sharp absorption peak could be observed in the $\mathrm{THz}$ spectrum of crystalline chlorobenzene samples (Fischer et al, 2007). The effects of water molecules in $\mathrm{THz}$ measurements were evidenced again by (Zhang \& Durbin, 2006), who reported significant differences in the $\mathrm{THz}$ absorption spectra of a myoglobin solution at various water concentrations, and (Liu \& Zhang, 2006), with investigation of D-glucose monohydrate with THz- TDS. Liu and co-workers showed that the absorption peak of D-glucose monohydrate decreases at a higher sample dehydration rate (Liu \& Zhang, 2006) as shown in Figure 3. 


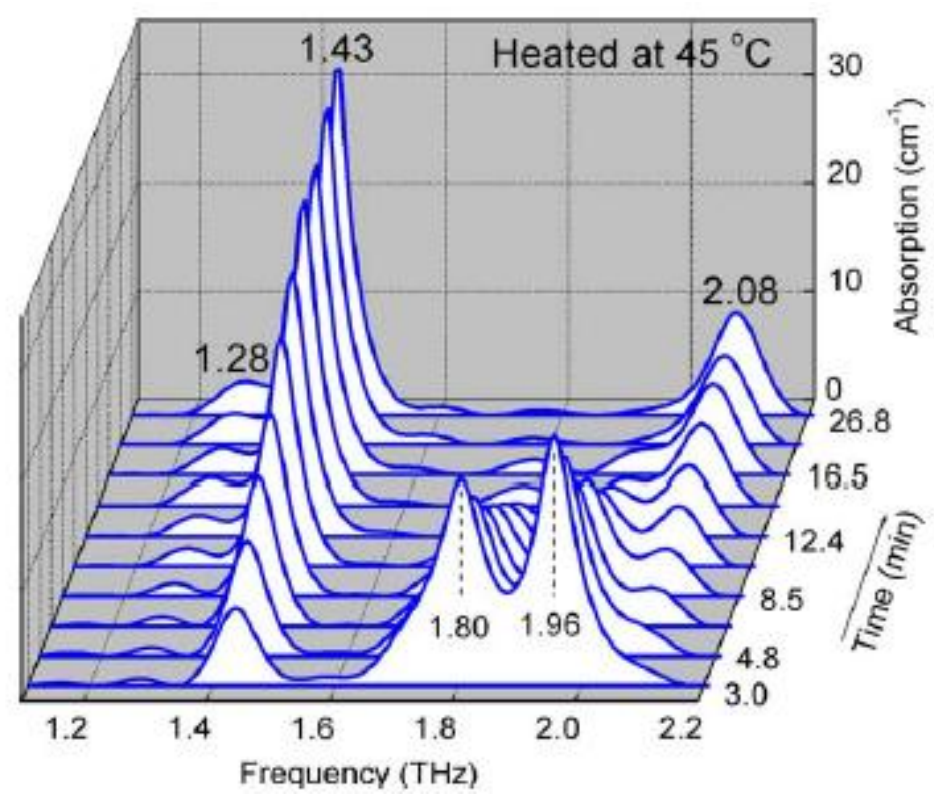

Figure 3. D-glucose monohydrate showcase escalating $\mathrm{THz}$ absorption peaks upon longer heating time, indicating that $\mathrm{THz}$ spectroscopy may be used as an analytical tool to study the dehydration of polycrystalline hydrate (Liu \& Zhang, 2006)

\section{THz SPECTROSCOPY AND INTERMO- LECULAR PROTEIN STRUCTURE}

The wide diversity in protein structure is matched by the vast variation in protein function in which each protein was specifically designed to cater to its role (Jackson \& Mantsch, 1995). The context in which biochemical processes occur in proteins is crucial to the thorough understanding of their structures and functions. The relationship between a protein's unique intermolecular structure and its corresponding function should be studied to gain a thorough view of proteins as a whole. $\mathrm{THz}$ spectroscopy is a relatively new method employed for studying intermolecular protein structure compared to other techniques, such as FTIR spectroscopy (Chirgadze \& Nevskaya, 1976), small-angle light scattering (Aymard et al, 1999), and Raman spectrometry (Clark et al, 1981). Investigations of biomolecular dynamics and structure can be greatly aided by a vibrational spectra study of the biomolecules at the $\mathrm{THz}$ frequency wavelength. The concerted motion of protein structure can be investigated by $\mathrm{THz}$ spectroscopy, which enables both energy and momentum transfer at the picosecond time scale. Comparison between the resolved THz spectra of the heme protein and its calculated vibrational modes showed that a relationship exists between the calculated structural collective modes and the hydration dependence of the absorption coefficient spectra (He et al, 2011a). Walther and co-workers investigated 3 different retinal isomers that exist in the photoactiveproteins rhodopsin, bacteriorhodopsin, and isorhodopsin of at $0.3-3 \mathrm{THz}(10-100$ $\mathrm{cm}^{-1}$ ) at $10 \mathrm{~K}$ and room temperature (Walther et al, 2000). The crystalline forms (prepared in polyethylene pellets) of these proteins differ only in their conformational structure. Clear differences between the 3 different retinal isomers' absorption spectra, specifically $1.2-2.1 \mathrm{THz}\left(40-70 \mathrm{~cm}^{-1}\right)$ at 10 $\mathrm{K}$, illustrate the capability of this spectral region to distinguish isomeric samples.

The ability of $\mathrm{THz}$ spectroscopy to probe into the intermolecular structure of biomolecules has attracted the attention of many research groups worldwide. Markelz and co-workers at the University of Connecticut investigated the ability of $\mathrm{THz}$ time domain spectroscopy (THz-TDS) to identify biomolecular species, conformational states, and mutations in bacteriorhodopsin (Markelz et al, 2002). Bacteriorhodopsin samples were subjected to various temperatures and environmental conditions to alter their architectural structures. The THz-TDS spectra of bacteriorhodopsin demonstrated remarkable differences before and after structural alternation (Markelz et al, 2002) at a frequency of 1-1.5 $\mathrm{THz}\left(33.3-50 \mathrm{~cm}^{-1}\right)$. The phenomena observed by 
Markelz and co-workers were probably due to conformational changes in the biomolecular structure correlating with a notable shift in the frequency spectrum (Markelz et al, 2002). This finding implies that the THz-TDS provides a non-destructive method for quantifying the intermolecular changes in biomolecules. It is also very apparent that THz-TDS has a high level of sensitivity to detect miniscule transformations in the conformation and mutation of biological systems.

The sensitivity of $\mathrm{THz}$ measurements to different $\beta$-pleated sheet orientations of crystalline tri- alanine and the effect of a solvate environment have been investigated by Siegrist (2006) at a frequency of $0.06-3 \mathrm{THz}\left(2 \mathrm{~cm}-1\right.$ to $\left.100 \mathrm{~cm}^{-1}\right)$. The X-ray structure obtained from the crystallography analysis of the anti-parallel tri-alanine showed a different structure of $\beta$-pleated sheet formation compared to that of parallel tri-alanine; this finding is in agreement with the $\mathrm{THz}$ spectra at $4.2 \mathrm{~K}$ where the spectra of parallel and anti-parallel tri-alanine showed distinctive spectral features (Siegrist et al, 2006). The finding by Siegrist and co-workers demonstrated the sensitivity of the $\mathrm{THz}$ spectral region for detecting conformational differences in secondary structures, the $\beta$-pleated sheet orientation in particular. The THz infrared absorption spectra lies in the range of $33.3-732.6 \mathrm{~cm}-1(1-22 \mathrm{THz})$, in which a dense set of absorption features can be ob- served from the measurements of solid-state amino acids in this region (Kutteruf et al, 2003).

The investigation of the hydration effect on a hen egg white lysozyme (HEWL) sample by $\mathrm{THz}$ spectroscopy has been conducted by Markelz and co-workers in the frequency wavelengths of 0.15 $1.95 \mathrm{THz}\left(5-65 \mathrm{~cm}^{-1}\right)$. A very similar study on the HEWL hydration rate was also conducted by Moeller and co-workers. In their study, they used farIR spectroscopy with a synchrotron light source in the frequency range of $0.5-1.35 \mathrm{THz}\left(15-45 \mathrm{~cm}^{-1}\right)$ (Moeller et al, 1992). Both studies have confirmed that at a higher temperature, an increase in the absorption intensities was observed due to high water absorption (Knab et al, 2006; Moeller et al, 1992). At a higher hydration rate, the absorption spectra of the lysozyme sample closely resembled that of pure water (Moeller et al, 1992). The confirmation of low-frequency sample vibrational motions was validated by calculations of a dielectric relaxation model (Knab et al, 2006). Recent study utilizing the $\mathrm{THz}$ wavelength that compares the spectra of HEWL at its natural and fibrillar state showed that the spectra of amorphous (HEWL in natural state) and long, elongated fibrils (HEWL in fibrillar state) have significant broad spectral features that might be due to the quaternary structure of the protein itself (Zakaria et al, 2011) as shown in Figure 4.

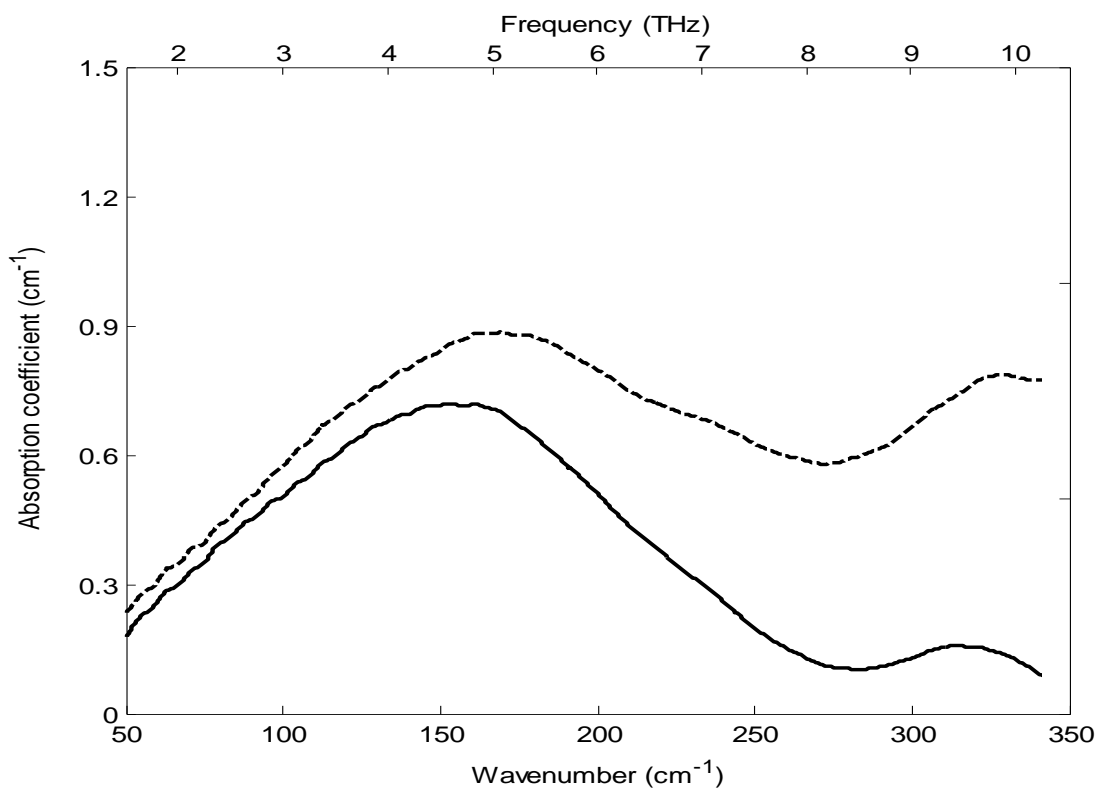

Figure 4: Absorption spectra of lysozyme fibril (__ _ $)$ and monomeric lysozyme (_ - ). Note that the spectra diverged after $150 \mathrm{~cm}^{-1}$ and onwards which indicate that the $\mathrm{THz}$ spectral region is able to differentiate the intra-molecular structures of proteins (Zakaria et al, 2011) 


\section{CONCLUSIONS}

Low frequency stretching and bending vibrations, crystalline phonon vibrations, hydrogen bond stretches and torsional vibrations of crystalline structures can be investigated in the $\mathrm{THz}$ wavelength (Beard et al, 2002). These attractive properties of $\mathrm{THz}$ spectroscopy couples with the fact that the non-ionizing, able to transmit through almost any materials of $\mathrm{THz}$ rays enable intra-molecular structure analysis in the low frequency spectral region, a spectral domain unachievable by other spectroscopic methods. There is an increasing interest in analysing low- frequency collective vibrational modes in biomolecules since these modes may provide information about their conformational state (Fischer et al, 2007). However there are several limitations in studying biomolecular structure in the $\mathrm{THz}$ range. The limitations are a stable and high energy light source connected to the spectrometer is necessary to enable analysis of protein structure in the very low frequency region which is unachievable in a conventional bench-top spectrometer. This bright light source may provide a higher signal-to-noise ratio and a wider dynamic range than traditional far-IR spectroscopy (Grischkowsky et al, 1990). The second factor to be considered was that the sample has to be rigorously characterized prior to spectroscopic measurements and it is best to measure the sample in pressed pellet form to ensure consistency in the resulting spectra. Protein samples immersed in water or solutions will induce interference to the spectra in the form of emergence of rotational water lines and poor signal-to-noise ratio.

\section{ACKNOWLEDGEMENTS}

I would like to thank Professor Anton Middelberg, Dr. Robert Falconer and Associate Professor Andrew Bradley for fruitful discussions towards the completion of this manuscript.

\section{REFERENCES}

1. Aymard P, Nicolai T, Durand D, Clark A (1999) Static and dynamic scattering of betalactoglobulin aggregates formed after heatinduced denaturation at $\mathrm{pH}$ 2. Macromolecules 32: 2542-2552

2. Bea S, Teich M (1974) Fundamentals of photonics, New York.
3. Beard MC, Turner GM, Schmuttenmaer CA (2002) Terahertz spectroscopy. Journal of Physical Chemistry B106: 7146-7159

4. Brandt NN, Chikishev AY, Kargovsky AV, Nazarov MM, Parashchuk OD, Sapozhnikov DA, Smirnova IN, Shkurinov AP, Sumbatyan NV (2008) Terahertz time-domain and Raman spectroscopy of the sulfur- containing peptide dimers: Low-frequency markers of disulfide bridges. Vibrational Spectroscopy 47: 53-58

5. Brown ER, Bjarnason JE, Chan TLJ, Lee AWM, Celis MA (2004) Optical attenuation signatures of Bacillus subtillis in the $\mathrm{THz}$ region. Applied Physics Letters 84: 3438-3440

6. Chirgadze YN, Nevskaya NA (1976) Infraredspectra and resonance interaction of amideone vibration of parallel-chain pleated sheet. Biopolymers 15: 627-636

7. Clark AH, Saunderson DHP, Suggett A (1981) Infrared and laser-Raman spectroscopic studies of thermally-induced globular protein gels. International Journal of Peptide and Protein Research 17: 353-364

8. Davies AG, Linfield EH, Johnston MB (2002) The development of terahertz sources and their applications. Physics in Medicine and Biology 47: 3679-3689

9. Doria A, Gallerano GP, Giovenale E, Messina G, Lai A, Ramundo-Orlando A, Sposato V, D’Arienzo M, Perrotta A, Romano M, Sarti M, Scarfi MR, Spassovsky I, Zeni O (2004) $\mathrm{THz}$ radiation studies on biological systems at the ENEA FEL facility. Infrared Physics \& Technology 45: 339-347

10. Dyakonov M, Shur M (1996) Detection, mixing, and frequency multiplication of terahertz radiation by two-dimensional electronic fluid. IEEE Trans Electron Devices 43: 380-387

11. Falconer RJ, Zakaria HA, Fan YY, Bradley AP, Middelberg AP (2010) Far-infrared spectroscopy of protein higher-order structures. Applied spectroscopy 64: 1259-1264

12. Fattinger C, Grischkowsky D (1989) TERAHERTZ BEAMS. Applied Physics 
Letters 54: 490-492

13. Ferguson B, Zhang XC (2002) Materials for terahertz science and technology. Nat Mater 1: 26-33

14. Fischer BM (2005a) Broadband THz TimeDomain Spectroscopy of Biomolecules, A Comprehensive Study of the Dielectric Properties of Biomaterials in the Far-Infrared, Doctor of Philosophy Thesis, Fakultat fur Mathematik und Physik, Universität Freiburg, Freiburg

15. Fischer BM, Helm H, Jepsen PU (2007) Chemical recognition with broadband $\mathrm{THz}$ spectroscopy. Proceedings of the Ieee 95: 1592-1604

16. Fischer BM, Hoffmann M, Helm H, Wilk R, Rutz F, Kleine-Ostmann T, Koch M, Jepsen PU (2005) Terahertz time-domain spectroscopy and imaging of artificial RNA. Optics Express 13: $5205-5215$

17. Giraud G, Karolin J, Wynne K (2003) Lowfrequency modes of peptides and globular proteins in solution observed by ultrafast OHDRIKES Spectroscopy. Biophys J 85: 1903-1913

18. Grischkowsky D, Keiding S, Vanexter M, Fattinger C (1990) Far-infrared timedomain spectroscopy with terahertz beams of dielectrics and semiconductors. Journal of the Optical Society of America B-Optical Physics 7: 2006-2015

19. Hagen E, Rubens H (1902) The reflection ability of some metals for ultraviolet and infrared radiation Ann Phys-Berlin 8: 1-21

20. He Y, Chen JY, Knab JR, Zheng W, Markelz AG (2011a) Evidence of Protein Collective Motions on the Picosecond Timescale. Biophysical Journal 100: 1058-1065

21. Jackson M, Mantsch HH (1995) The use and misuse of FTIR spectroscopy in the determination of protein-structure. Critical Reviews in Biochemistry and Molecular Biology 30: 95-120

22. Kachorovskii VY, Shur MS (2008) Field effect transistor as ultrafast detector of modulated terahertz radiation. Solid-State Electron 52: 182-185

23. Knab J, Chen JY, Markelz A (2006) Hydration dependence of conformational dielectric relaxation of lysozyme. Biophys $J$ 90: 25762581

24. Knap W, Dyakonov MI (2013) 5 - Field effect transistors for terahertz applications. In Handbook of Terahertz Technology for Imaging, Sensing and Communications, Saeedkia D (ed), pp 121-155. Woodhead Publishing

25. Kutteruf MR, Brown CM, Iwaki LK, Campbell MB, Korter TM, Heilweil EJ (2003) Terahertz spectroscopy of short-chain polypeptides. Chemical Physics Letters 375: 337-343

26. Liu HB, Zhang XC (2006) Dehydration kinetics of D-glucose monohydrate studied using $\mathrm{THz}$ time-domain spectroscopy. Chemical Physics Letters 429: 229-233

27. Lu JQ, Shur MS, Hesler JL, Sun LQ, Weikle R (1998) Terahertz detector utilizing twodimensional electronic fluid. IEEE Electron Device Lett 19: 373-375

28. Markelz A, Whitmire S, Hillebrecht J, Birge $\mathrm{R}$ (2002) THz time domain spectroscopy of biomolecular conformational modes. Physics in Medicine and Biology 47: 3797-3805

29. Markelz AG (2008) Terahertz dielectric sensitivity to biomolecular structure and function. Ieee Journal of Selected Topics in Quantum Electronics 14: 180-190

30. Mickan SP, Menikh A, Liu HB, Mannella CA, MacColl R, Abbott D, Munch J, Zhang XC (2002) Label-free bioaffinity detection using terahertz technology. Physics in Medicine and Biology 47: 3789-3795

31. Moeller KD, Williams GP, Steinhauser S, Hirschmugl C, Smith JC (1992) HYDRATION-DEPENDENT FARINFRARED ABSORPTION IN LYSOZYME DETECTED USING SYNCHROTRON RADIATION. Biophys $J$ 61: 276-280 
32. Pickwell E, Cole BE, Fitzgerald AJ, Wallace VP, Pepper M (2004) Simulation of terahertz pulse propagation in biological systems. Applied Physics Letters 84: 21902192

33. Picometrix.(2005)T-RAY2000 ANALYTICAL SYSTEM HARDWARE MANUAL. Picometrix,Inc., Michigan,US. Plusquellic DF, Siegrist K, Heilweil EJ, Esenturk M (2007) Applications of terahertz spectroscopy in biosystems. Chemphyschem 8: 2412-2431

34. Png GM, Falconer RJ, Fischer BM, Zakaria HA, Mickan SP, Middelberg AP, Abbott D (2009) Terahertz spectroscopic differentiation of microstructures in protein gels. Optics express 17: 13102-13115

35. Sauer K (1995) WHY SPECTROSCOPY WHICH SPECTROSCOPY. In Biochemical Spectroscopy Vol. 246, pp 1-10. San Diego: Academic Press Inc

36. Shen YC, Upadhya PC, Linfield EH, Beere HE, Davies AG (2003) Ultrabroadband terahertz radiation from low-temperature-grown GaAs photoconductive emitters. Applied Physics Letters 83: 3117-3119

37. Siegrist K, Bucher CR, Mandelbaum I, Walker ARH, Balu R, Gregurick SK, Plusquellic DF (2006) High- resolution terahertz spectroscopy of crystalline trialanine: Extreme sensitivity to beta-sheet structure and cocrystallized water. $J$ Am Chem Soc 128: 5764-5775

38. Smye SW, Chamberlain JM, Fitzgerald AJ, Berry E (2001) The interaction between Terahertz radiation and biological tissue. Physics in Medicine and Biology 46: R101-R112

39. Trzebiatowska-Gusowska M, Plinski EF, Baran J, Walczakowski MJ, Jarzab PP, Nowak K, Fuglewicz B, Mikulics M, Palka N, Szustakowski M (2014) Terahertz and Raman spectra of non-centrosymmetrical organic molecular crystals. Optical Materials 37: 28-35
40. Ueno Y, Ajito K (2008) Analytical terahertz spectroscopy. Analytical Sciences 24: 185-192

41. Vanexter M, Grischkowsky D (1990) Optical and Electronic-Properties of Doped Silicon From 0.1 To 2 Thz. Applied Physics Letters 56: 1694-1696

42. Walther M, Fischer B, Schall M, Helm H, Jepsen PU (2000) Far-infrared vibrational spectra of all-trans, 9- cis and 13-cis retinal measured by $\mathrm{THz}$ time-domain spectroscopy. Chemical Physics Letters 332: 389-395

43. Walther M, Plochocka P, Fischer B, Helm H, Jepsen PU (2002) Collective vibrational modes in biological molecules investigated by terahertz time-domain spectroscopy. Biopolymers 67: 310-313

44. Woodward RM, Wallace VP, Arnone DD, Linfield EH, Pepper M (2003) Terahertz pulsed imaging of skin cancer in the time and frequency domain. Journal of Biological Physics 29: 257-261

45. Yamaguchi M, Miyamaru F, Yamamoto K, Tani M, Hangyo M (2005) Terahertz absorption spectra of L-, D-, and DL-alanine and their application to determination of enantiometric composition. Applied Physics Letters $\mathbf{8 6}$

46. Yin XX, Kong KM, Lim JW, Ng BWH, Ferguson B, Mickan SP, Abbott D (2007) Enhanced T-ray signal classification using wavelet preprocessing. Medical \& Biological Engineering \& Computing 45: 611-616

47. Zakaria HA, Fischer BM, Bradley AP, Jones I, Abbott D, Middelberg AP, Falconer RJ (2011) Low-frequency spectroscopic analysis of monomeric and fibrillar lysozyme. Applied spectroscopy 65: 260-264

48. Zhang CF, Durbin SM (2006) Hydrationinduced far-infrared absorption increase in myoglobin. Journal of Physical Chemistry $B$ 110: $23607-2361$ 